\title{
Commissioning of the Cylindrical Drift Chamber for the COMET experiment
}

\author{
Manabu Moritsu* \\ Institute of Particle and Nuclear Studies (IPNS), High Energy Accelerator Research \\ Organization (KEK), Tsukuba 305-0801, Japan \\ E-mail: moritsudpost.kek.jp
}

Yoshitaka Kuno, Yugo Matsuda, Yu Nakazawa, Saki Ohta, Hideyuki Sakamoto†, Akira Sato, Ming Liang Wong

Department of Physics, Osaka University, Toyonaka 560-0043, Japan

\section{Xiaoshan Jiang, Hai-Bo Li, Yohei Nakatsugawa, Jie Zhang}

Institute of High Energy Physics (IHEP), Chinese Academy of Sciences, Beijing 100049, China

\begin{abstract}
A cylindrical drift chamber (CDC) has been developed for the COMET Phase-I experiment. To search for the muon-to-electron conversion with a sensitivity of $3 \times 10^{-15}$, the CDC is required to have momentum resolution of $200 \mathrm{keV} / \mathrm{c}$ for $100-\mathrm{MeV} / \mathrm{c}$ electrons. In order to reduce the multiple scattering, we have selected a chamber gas mixture of $90 \%$ helium with $10 \%$ isobutane. Construction of the CDC was successfully conducted, and performance evaluation is being carried out. We have confirmed stable operation without a magnetic field thus far based on cosmic-ray tests. In this paper, the motivation, design, construction, and commissioning status of the CDC are presented.
\end{abstract}

European Physical Society Conference on High Energy Physics - EPS-HEP2019 -

10-17 July, 2019

Ghent, Belgium

\footnotetext{
* Speaker.

${ }^{\dagger}$ Present address: Center for Computational Science, RIKEN.

‡Present address: Université Clermont Auvergne.
} 


\section{Introduction}

The COMET experiment aims to search for the neutrinoless coherent transition of a muon to an electron in the field of an aluminum nucleus, $\mu^{-} N \rightarrow e^{-} N$, which violates the lepton flavor conservation and has never been observed yet thus far [ [U, [ []]. The process is extremely suppressed in the Standard Model even if the neutrino oscillation effect is taken into account. However, the conversion rate is predicted to be enhanced in most of well-motivated new physics models beyond the Standard Model. The goal of the COMET Phase-I is to explore the muon-to-electron conversion with a single event sensitivity of $3 \times 10^{-15}$, which is 100 times better than the current limit.

The experiment takes place at J-PARC with a high-quality pulsed beam and unprecedentedly powerful muon source. To search for the muon-to-electron conversion, negative muons are stopped in a target material and form a muonic atom with a nucleus. Within the Standard Model, fate of the muon is either muon decay in orbit (DIO) or nuclear muon capture with fractions of $39 \%$ and $61 \%$, respectively for aluminum nucleus. Then, if the muon-to-electron conversion happens, we could observe an electron without neutrinos. Since the process is coherent, the emitted electron has a unique energy of $105 \mathrm{MeV}$ for aluminum nuclei.

Whilst the signal of the muon-to-electron conversion is the mono-energetic $105 \mathrm{MeV}$ electron, one of the significant backgrounds is a high-energy tail of the DIO electron distribution [3]. In order to distinguish the signal from the DIO background, good resolution of $200 \mathrm{keV} / \mathrm{c}$ is required in terms of the electron momentum measurement.

\section{Cylindrical Drift Chamber}

In the COMET Phase-I experiment, the converted electrons are detected with a cylindrical drift chamber (CDC) and trigger hodoscopes in a solenoidal magnetic field of $1 \mathrm{~T}$. Figure $\mathrm{W}$ shows
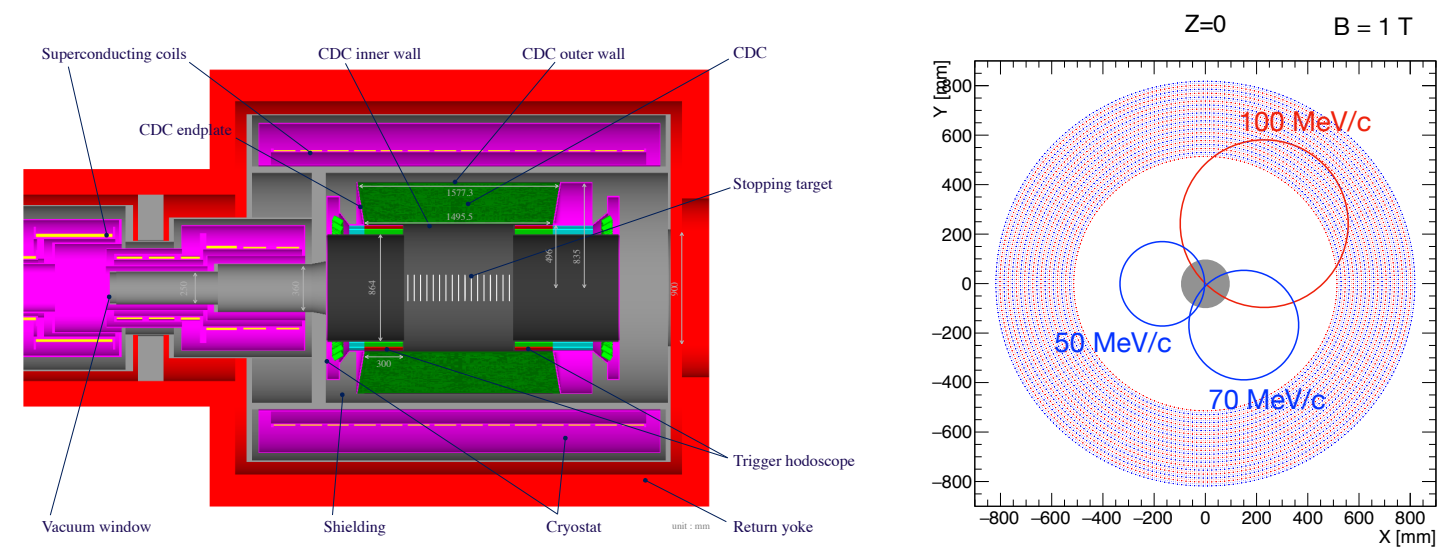

Figure 1: Schematic layout of the Cylindrical Detector System for the COMET Phase-I experiment. Muon beams come from the left side.

Figure 2: Cross-sectional view of the $\mathrm{CDC}$ at the center of longitudinal direction $(Z=0)$. Circles indicate trajectories of charged particles with corresponding momenta at a $1 \mathrm{~T}$ magnetic field. 
a schematic layout of the Cylindrical Detector System. Muons, coming from the upstream, are stopped in aluminum target discs placed in the center of the detector solenoid, and transverselyemitted electrons are measured by the Cylindrical Detector System.

The CDC has a large inner diameter of approximately $1 \mathrm{~m}$, because of the following two reasons. First, since the detector area is exposed to the beam flash at a prompt beam timing, the detectors have to avoid large-emittance beam stream. The second reason is to suppress the DIOelectron hits. The acceptance of the CDC is designed to open from around $70 \mathrm{MeV} / \mathrm{c}$ as illustrated in Fig. ․ Most of the DIO-electrons have less than $50 \mathrm{MeV} / \mathrm{c}$ momenta, and therefore do not reach the CDC, which is quite helpful to mitigate the CDC hit occupancy.

Specifications of the CDC is listed in Table $\mathbb{W}$. The inner and outer cylinders are made of carbon-fiber-reinforced plastic (CFRP) with thicknesses of 0.5 and $5.0 \mathrm{~mm}$, respectively. The endplates are made of 10-mm aluminum with slightly tapered shape to sustain a wire tension load. The number of layers are 18 with 2 guard layers as the innermost and outermost layers. An alternated all stereo layer configuration with $\pm(64-75)$-mrad tilt angles is designed to achieve good spatial resolution for the longitudinal direction. A partial cross section of the CDC cell structure is shown in Fig. [l. Although the cell shapes are slightly distorted according to their positions, the typical single cell geometry is almost square with $16.0-\mathrm{mm}$ height and $16.8-\mathrm{mm}$ width as shown in Fig. .

In order to accomplish the required momentum resolution of $200 \mathrm{keV} / \mathrm{c}$ for the $105-\mathrm{MeV} / \mathrm{c}$ electrons, the $\mathrm{CDC}$ is designed to reduce material thickness to suppress the multiple scattering

Table 1: Specifications of the CDC [D]].

\begin{tabular}{lll}
\hline \hline Inner wall (CFRP) & Length & $1495.5 \mathrm{~mm}$ \\
& Radius & $496.0-496.5 \mathrm{~mm}$ \\
& Thickness & $0.5 \mathrm{~mm}$ \\
\hline Outer wall (CFRP) & Length & $1577.3 \mathrm{~mm}$ \\
& Radius & $835.0-840.0 \mathrm{~mm}$ \\
& Thickness & $5.0 \mathrm{~mm}$ \\
\hline Number of layers & Sense layer & 18 \\
& Guard layer & 2 \\
\hline Stereo angle & & $\pm(64-75) \mathrm{mrad}$ \\
\hline Cell size & Height & $16.0 \mathrm{~mm}$ \\
& Width & $16.8 \mathrm{~mm}$ \\
\hline Sense wire & Material & $($ Au-plated) W \\
& Diameter & $25 \mu \mathrm{m}$ \\
& Number of wires & 4,986 \\
\hline Field wire & Material & $($ unplated) Al \\
& Diameter & $126 \mu \mathrm{m}$ \\
& Number of wires & 14,562 \\
\hline Gas & Mixture & He:iC ${ }_{4} \mathrm{H}_{10}(90: 10)$ \\
& Volume & $2,084 \ell$ \\
\hline \hline
\end{tabular}




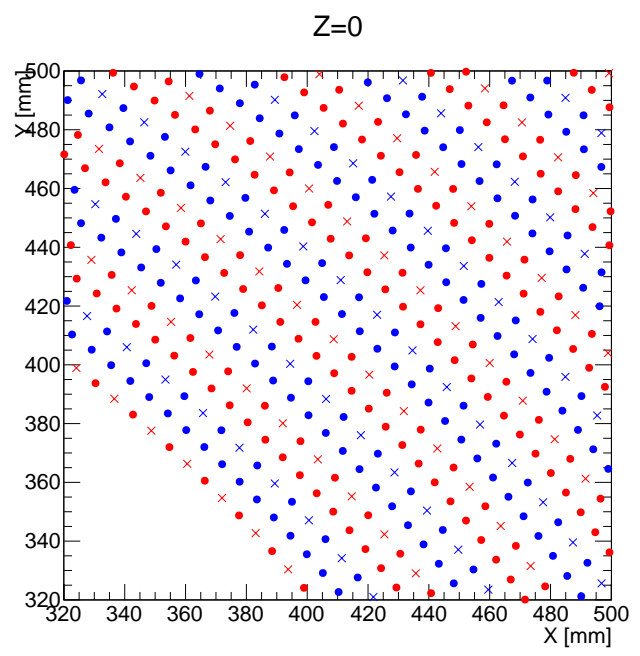

Figure 3: A partial cross section of the CDC cell structure. The crosses and circles indicate sense and field wires. The red and blue colors represent positive and negative stereo angles.

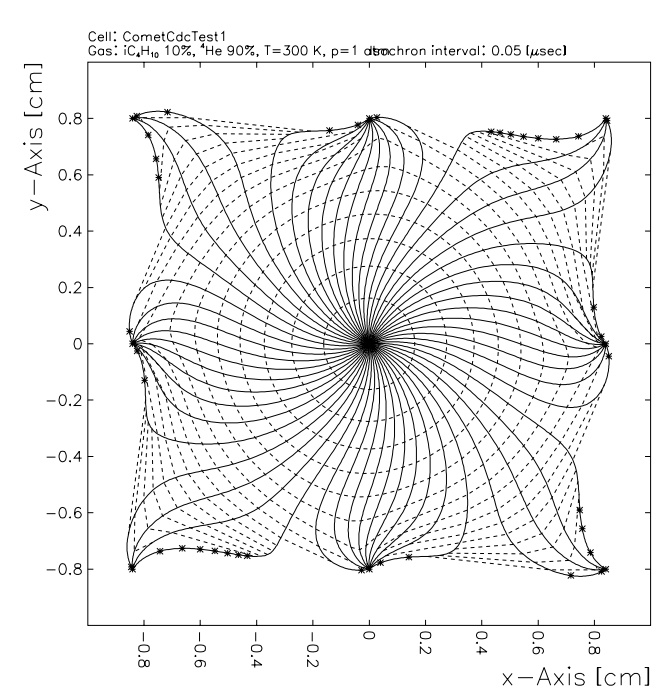

Figure 4: Typical single cell layout with drift lines and isochrones of 50-ns intervals at 1$\mathrm{T}$ magnetic field with a sense wire voltage of $1800 \mathrm{~V}$.

Table 2: Properties of the $\mathrm{He}: \mathrm{iC}_{4} \mathrm{H}_{10}(90: 10)$ gas mixture at $20^{\circ} \mathrm{C}$ and $1 \mathrm{~atm}$. Values of energy loss, primary ions, and total ions are for minimum ionizing particles, computed from [四]. Values of drift velocity and longitudinal diffusion are at 1000-V/cm electric field, obtained from [1], 回].

\begin{tabular}{ll}
\hline \hline Parameter & Value \\
\hline Mixture $\mathrm{He}: \mathrm{iC}_{4} \mathrm{H}_{10}$ & $90: 10$ \\
Radiation length & $1400 \mathrm{~m}$ \\
Energy loss & $0.88 \mathrm{keV} / \mathrm{cm}$ \\
Primary ions & $13.5 / \mathrm{cm}$ \\
Total ions & $29.5 / \mathrm{cm}$ \\
Drift velocity & $\sim 2.2 \mathrm{~cm} / \mu \mathrm{s}$ \\
Longitudinal diffusion & $\sim 160 \mu \mathrm{m}$ for $1-\mathrm{cm}$ drift \\
\hline \hline
\end{tabular}

which dominates momentum resolution in this low energy region. Hence, $90 \%$ helium with a quencher of $10 \%$ isobutane is selected as the chamber gas mixture. Properties of the $\mathrm{He}: \mathrm{iC}_{4} \mathrm{H}_{10}$ (90:10) gas mixture is summarized in Table $\square$. Since low- $Z$ materials have a small photoelectric cross section, they have an additional advantage to reduce background hits caused by low-energy photons from the muon stopping target.

\section{Construction of the CDC}

The aluminum endplates and the CFRP outer cylinder were fabricated before wire stringing. Wire feedthrough holes were precisely drilled on the endplates with tolerances of $0.05 \mathrm{~mm}$ for the position and $0.01 \mathrm{~mm}$ for the diameter. Wire stringing for the 19,548 total wires was per- 


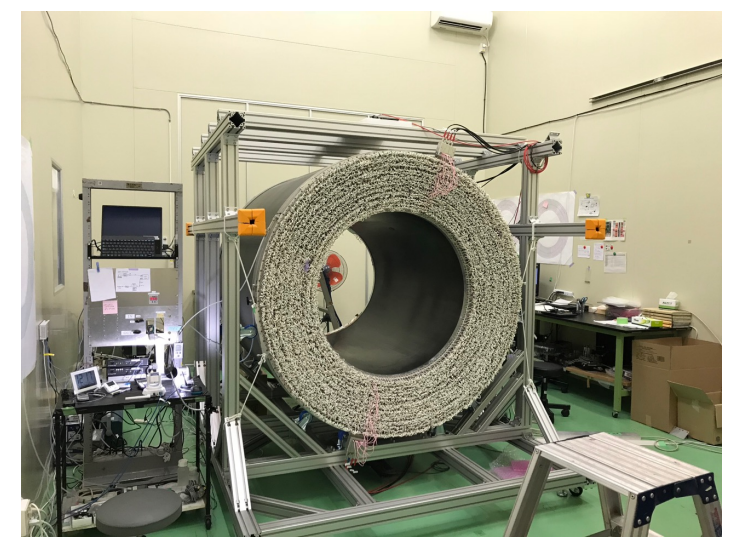

Figure 5: Photograph of the CDC taken from the upstream side.

formed after attaching the endplates with the outer cylinder. We use gold-plated tungsten wires with $25-\mu \mathrm{m}$ diameter for the sense wires, and unplated aluminum wires with $126-\mu \mathrm{m}$ diameter for the field wires. During the whole period of stringing, the room conditions were kept within 19.5$23.5^{\circ} \mathrm{C}$ temperature and $30-55 \%$ relative humidity. To compensate an estimated total wire tension of 1.4 ton, a pre-stress were applied between the endplates in advance and released appropriately as the wires were strung from outside to inside. The wires are fixed at feedthrough pipes with climping. The nominal tension weights for the sense and field wires were set to be 50 and $80 \mathrm{~g}$, which correspond to the gravitational sags of 50 and $120 \mu \mathrm{m}$ at the center, respectively. We confirmed that the sag for the sense wires was controlled between 48 and $61 \mu \mathrm{m}$, and the sag differences between the sense and surrounding field wires are kept within less than $100 \mu \mathrm{m}$ [ $[\mathbf{0}]$.

The 0.5-mm thick CFRP inner cylinder was installed after the wire stringing to close the gas volume of 2,084 litters. The gas tightness was checked with a helium gas, and leak points were sealed with silicone gum. Figure 5 shows a photograph of the CDC after completion.

\section{Readout Electronics}

We use readout frontend electronics boards developed for the Belle-II CDC [8] with appropriate modifications. Each board has 48 input channels, 6 amplifier-shaper-discriminator chips, 6 ADCs and an FPGA. The ADC provides 10-bit pulse-height information with a 30-MHz sampling. The TDC function is implemented in the FPGA with a 960-MHz digitization. In order to withstand a severe radiation environment in the COMET Phase-I experiment, the FPGA firmware is developed with an auto-recovery scheme tolerable against $10^{12} \mathrm{~cm}^{-2}$ for $1-\mathrm{MeV}$ equivalent neutron fluence [Q].

\section{Performance Test}

CDC performance tests using cosmic rays are being carried out. Stable operation of the CDC was established with the $\mathrm{He}: \mathrm{iC}_{4} \mathrm{H}_{10}$ (90:10) gas mixture and with an applied high voltage up to $1850 \mathrm{~V}$. Figure (a) shows a residual distribution obtained from the deviation of drift distance from 
(a) Residual distribution

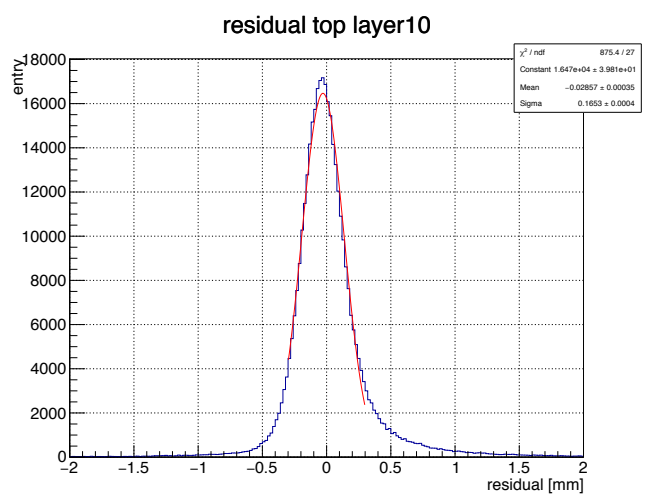

(b) Spatial resolution vs DCA

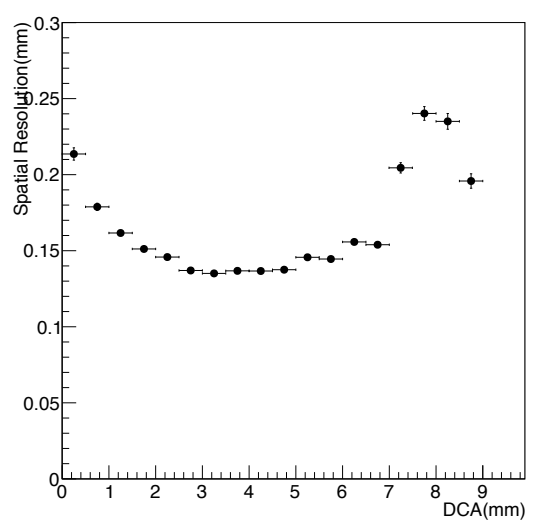

Figure 6: (a) Residual distribution for a central layer at $1825 \mathrm{~V}$. The red line denotes a Gaussian fitting result of the core part. (b) Spatial resolution versus distance of closest approach (DCA) between a hit wire and a reconstructed track. The spacial resolution here includes a tracking uncertainty.

the distance of closest approach (DCA) between a hit wire and a reconstructed track. The spatial resolution was extracted from the residual distribution by fitting tracks excluding one layer, and estimated to be less than $170 \mu \mathrm{m}$ at central layers. Note that the resolution here includes a tracking uncertainty. Figure G(b) shows a spatial resolution versus DCA. While the resolution is smaller than $140 \mu \mathrm{m}$ around a middle range of the DCA, it tends to be larger near the sense wire and field wires. A relatively small number of primary ions (see Table $\mathbb{Z}$ ) deteriorates a spatial resolution in the small DCA region, and a distortion of the electric field near the field wires affects the resolution in the cell edge region. Hit efficiency is estimated to be higher than $98 \%$.

We have demonstrated that the CDC shows a decent performance as expected, and will proceed to detailed evaluation in a magnetic field after construction of the detector solenoid magnet.

\section{References}

[1] Y. Kuno (COMET Collaboration), Prog. Theor. Exp. Phys. 2013, 022 C01 (2013).

[2] R. Abramishvili et al. (COMET Collaboration), arXiv:1812.09018, to be published in Prog. Theor. Exp. Phys.

[3] A. Czarnecki, X.G. i Tormo, and W.J. Marciano, Phys. Rev. D 84, 013006 (2011).

[4] S. Tavernier, Experimental Techniques in Nuclear and Particle Physics, Springer-Verlag 2010.

[5] Garfield simulation, https://garfield.web.cern.ch/garfield.

[6] A. Sharma and F. Sauli, Nucl. Instrum. and Methods A 350, 470 (1994).

[7] M. Moritsu et al., PoS ICHEP2018 340, 538 (2019).

[8] T. Uchida et al., IEEE Nucl. Sci. Symp. Med. Imag. Conf. 2011, pp.694-698 (2011).

[9] Y. Nakazawa et al., Nucl. Instrum. and Methods A 936, 351 (2019). 